\title{
SUPOSIÇÃO DE NORMALIDADE E GESTÃO DE RISCO: UMA APLICAÇÃO DO VAR PARAMÉTRICO VIA TESTE DE ADERÊNCIA
}

\section{NORMALITY ASSUMPTIONS AND RISK MANAGEMENT: AN APPLICATION OF THE PARAMETRIC VAR VIA GOODNESS-OF-FIT TEST}

\author{
Herick Fernando Moralles* E-mail: herick@sc.usp.br \\ Alexandre Sartoris Neto** E-mail: asartoris@uol.com.br \\ Daisy Aparecida do Nascimento Rebelatto* E-mail: daisy@usp.sc.br \\ *Universidade de São Paulo (USP), São Paulo, SP \\ **Universidade estadual Júlio de Mesquita filho (UNESP), São Paulo, SP
}

\begin{abstract}
Resumo: Tendo em vista as fragilidades do VaR (Valor-em-Risco) paramétrico com pressuposto de normalidade, este trabalho desenvolve um método de cômputo do VaR paramétrico ajustado para dez diferentes distribuições de probabilidade. Especificamente, a distribuição a ser utilizada no cálculo do VaR para um dado ativo ou carteira é apontada pelo teste de aderência de Kolmogorov-Smirnov. Ainda, a investigação compara a aplicabilidade da suposição de normalidade para o cálculo do VaR para ativos individuais, e uma grande carteira, num contexto de estabilidade de mercados. $\mathrm{O}$ experimento realizado faz uso de uma amostra de 15 ativos individuais negociados na Bolsa de Valores de São Paulo, juntamente com o índice IBOVESPA, coletados na base Economática ${ }^{\circledR}$. Os testes de aderência e os cálculos do VaR são realizados por um programa desenvolvido em MATLAB $7.1 \circledR$. É encontrada uma grande ocorrência de ativos com boa aderência à distribuição Normal, demonstrando que o pressuposto de normalidade traz boas estimativas de risco tanto para um grande portfólio quanto para ativos individuais.
\end{abstract}

Palavras-Chave: VaR (Valor-em-Risco) paramétrico. Normalidade. Kolmogorov-Smirnov.

Abstract: Given the weaknesses of the parametric $\mathrm{VaR}$ (Value-at-Risk) calculated by normality assumptions, this paper develops a method of parametric VaR calculation considering ten different probability distributions. Specifically, the distribution to be used for the VaR calculation of a specific asset or portfolio is indicated by the Kolmogorov-Smirnov goodness-of-fit test. Additionally, the study compares the normality assumptions applicability for the VaR calculation of both individual assets, and to a large portfolio, in the context of market stability. The experiment makes use of a sample of 15 individual assets traded in the Sao Paulo Stock Exchange and the IBOVESPA index, collected in the Economática ${ }^{\circledR}$ database. The goodness-of-fit tests and VaR calculations are performed by a program developed in MATLAB7.1®. This investigation demonstrates that the assumption of normality brings good risk estimates for large portfolios and individual assets.

Keywords: Parametric VaR (Value-at-Risk). Normality. Kolmogorov-Smirnov.

\section{INTRODUÇÃO}

A teoria financeira aceita de forma ampla a variância dos possíveis resultados como medida de risco, sendo possível atribuir um número a um conceito tratado de 
forma qualitativa. Contudo, nos anos noventa, foi desenvolvido o método VaR (Valorem-Risco), capaz de medir o risco de um portfólio, resumindo em um único número tal exposição às perdas. Tal facilidade de cálculo e interpretação fez com que o VaR fosse amplamente utilizado (MARQUES et al., 2001; JORION, 1997).

A crescente evolução dos mercados financeiros, ao lado do grande volume de ativos negociados, faz com que a mensuração do risco seja a principal preocupação dos gestores, visto que os instrumentos de avaliação de risco possuem um papel crucial no desempenho de instituições financeiras, e conseqüentemente em sua capacidade de honrar compromissos.

Tal panorama se tornou ainda mais preponderante na recente crise financeira, em que uma série de instituições finaceiras faliram ou foram salvas por seus governos, como Lehman Brothers, AIG e Royal Bank of Scotland, exacerbando ainda mais a importância de ferramentas confiáveis para a gestão de risco (MABROUK; SAADIB, 2012).

O cômputo do VaR tem se tornado de suma importância para a gestão de risco. De fato, a principal implicação da criação do comitê da Basiléia para supervisão bancária era que instituições financeiras com sistemas de gestão de risco suficientemente desenvolvidos, tivessem o VaR como ferramenta padrão para mensuração do risco e necessidade de reservas de capital (ESCANCIANO; OLMO, 2010).

Contudo, o VaR e suas diferentes formas de cálculo possuem uma série de fragilidades, e atualmente, os modelos de cálculo de VaR tem se tornado cada vez mais complexos em termos matemáticos e computacionais, especialmente no âmbito do VaR calculado via teoria dos valores extremos e modelos autoregressivos de heteroscedasticidade condicional (GARCH).

Modelos paramétricos de VaR em sua maioria fazem uso de pressupostos de normalidade para os retornos dos ativos, o que pode causar subestimação da exposição ao risco, devido à conhecida natureza leptocúrtica dos dados financeiros (KAWATA; KIJIMA, 2007).

A suposição de normalidade é ainda mais utilizada para carteiras grandes e diversificadas, visto que a distribuição dos retornos da carteira será o somatório das distribuições dos retorno dos ativos individuais da carteira, resultando assim, em 
uma tendencia ao comportamento gaussiano.

A fim de tornar o cômputo do VaR mais preciso e simultaneamente simples em termos de compelxidade matemática e computacional, bem como excluindo pressupostos distribucionais, o presente trabalho propõe um método de cálculo do VaR paramétrico baseado em distribuições de probabilidade resultantes do teste de aderência de Kolmogorov-Smirnov.

Adicionalmente, intenta-se verificar a aplicabilidade da suposição de normalidade para o cálculo do VaR tanto para ativos individuais, quanto para uma grande carteira, num contexto de estabilidade de mercados.

Dessa forma, este estudo visa identificar empiricamente a distribuição de probabilidade do índice IBOVESPA e de algumas ações de empresas individuais, de pequeno, médio e grande porte com ações negociadas na Bolsa de Valores de São Paulo. A partir disso calcula-se o risco destes ativos por meio do VaR paramétrico, tendo em vista a melhor distribuição de probabilidade teórica encontrada após a realização do teste de aderência de Kolmogorov-Smirnov, realizado com 10 diferentes distribuições de probabilidade.

Portanto, a distribuição empírica observada (discreta) será transformada em uma distribuição contínua para o cálculo do VaR paramétrico. Dessa forma, será testada a eficácia do pressuposto de normalidade tanto para uma carteira grande e diversificada como o índice IBOVESPA, quanto para ativos individuais num contexto de relativa estabilidade econômica.

\section{REVISÃO DE LITERATURA}

\subsection{Valor Em Risco (VaR)}

Atualmente, uma metodologia muito usada para a avaliação de risco financeiro em portfolios é o $\mathrm{VaR}$, o qual é uma medida probabilística que ganhou tamanha popularidade em função de sua simplicidade de cálculo, e a capacidade de resumir em apenas um número o risco de uma carteira de ações (JORION, 1997).

O VaR é a perda máxima esperada de uma carteira, a um nível de significância $\alpha$ (ou nível de confiança $1-\alpha$ ), em um horizonte temporal 
determinado. A título de exemplo, o J.P.Morgan utiliza um nível de confiança de 95\% para um horizonte temporal de 20 dias (JORION, 1997). Sendo assim, a expectativa é de que a cada 20 dias haja uma perda que supere o VaR. (JORION, 1997; SOUZA, 1999).

Assim, o VaR pode ser expressado pela Equação 1.

$$
\operatorname{Pr}[\Delta P(\tau)<-V a R]=1-c
$$

Onde,

$\Delta P(\tau)$ é a variação percentual dos retornos.

$c$ é o nível de confiança.

A Equação (1) contempla a idéia geral da teoria que cerne o VaR. Contudo, sua forma de cálculo não é única, existindo dentre outros, três principais categorias de cálculo para o VaR: Não-paramétrico, e Semi-paramétrico, Paramétrico.

Segundo (Wang et. al. 2010) dentre os métodos não-paramétricos estão os métodos de simulação histórica e simulação de Monte-Carlo. O método de simulação histórica utiliza a distribuição empírica dos retornos sem utilizar qualquer pressuposição probabilística, utilizando assim, o passado para prever o futuro. Obviamente este método é falho em considerar que os retornos futuros não irão se modificar.

Já o método da simulação de Monte-Carlo assume uma distribuição de probabilidade qualquer para simular os retornos de um ativo ou portfólio específico. (JORION, 1997). Assim, tal método é falho no que tange à escolha da distribuição de probabilidade correta

Quanto aos métodos semi-paramétricos, encontra-se especialmente a teoria dos valores extremos que estuda a perda na cauda da distribuição, diminuindo assim a importância dos valores centrais (WANG et. al. 2010).

O principal método de cálculo do VaR paramétrico, objeto do presente estudo, é conhecido como método da variância-covariância. Aqui, é assumida uma distribuição de probabilidade específica para o cômputo do VaR, que segundo (Wang et. al. 2010) pode ser expresso pela Equação 1.

$$
\operatorname{VaR}_{t}(\alpha)=\mu_{t}+\phi_{t}^{-1}(\alpha) \sigma_{t}
$$

Onde, 
$\mu$ e $\sigma$ são os parâmetros de uma determinada distribuição de probabilidade $\phi(\cdot)$.

$\mathrm{Na}$ realidade, o VaR paramétrico pode ser calculado a partir da cauda esquerda da distribuição de probabilidade dos retornos do portfólio ou ativo individual, considerando que não existam grandes mudanças nas condições do mercado em questão. Não obstante, tal modelo é usualmente empregado com alternativas de estimação da volatilidade dos retornos, como ocorre nos modelos de VaR cujo desvio-padrão é estimado via modelos autoregressivos de heteroscedasticidade condicional (KHINDANOVA, 2001; AUSSENEGG e MIAZHYNSKAIA, 2006).

A título de exemplo, caso seja pressuposto que os retornos de um ativo sigam uma distribuição Normal, o cálculo do VaR se resume em calcular o $(1-\mathrm{c})$ percentil da distribuição Normal, que pode ser visualizada segundo Khindanova (2001) em (3).

$$
1-c=\int_{-\infty}^{z-c} \phi(z) d z
$$

Onde,

$\phi(z)$ é a função densidade de probabilidade de uma distribuição Normal padronizada, seguindo a idéia de que os retornos do ativo sejam normalmente distribuídos.

Assim, os dados do ativo serão utilizados para calcular os parâmetros da distribuição em questão, e o VaR será calculado com base nos parâmetros de tal distribuição. Portanto, a eficiência do VaR paramétrico está intimamente relacionada com a distribuição de probabilidade da variável a ser analisada, a qual terá grande impacto na acurácia da exposição ao risco estimada.

Contudo, o computo do VaR, muitas vezes, é feito de modo a pressupor normalidade, especialmente para portfolios grandes e diversificados como afirma Jorion (1997), com o objetivo de simplificar seu cálculo.

Tal simplificação pode levar a subestimação ou sobre-estimação do risco, caso seja considerada uma distribuição de probabilidade errônea, como demonstrado na FIGURA 1, que ilustra duas distribuições contínuas com diferentes caudas esquerdas. Nesse caso, é claramente observável a subestimação do risco 
de um ativo que possui distribuição de Gumbel (Valores extremos à esquerda), quando suposta a normalidade desse ativo (BARBACHAN et al. 2006; RAMANATHAN, 1993; GUJARATI, 2000; KLEIBER \& KOTZ, 2003).

Figura 1 - Diferença nas caudas de distribuições distintas

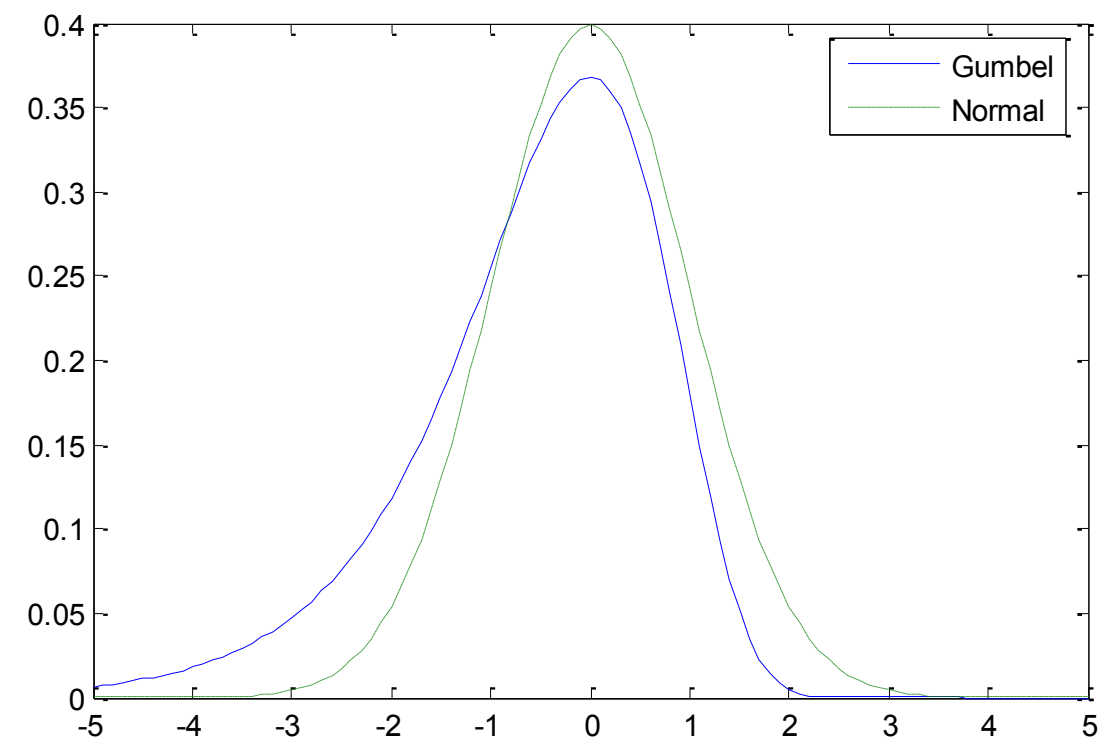

(Gráfico confeccionado em MATLAB®)

Especificamente é necessária uma comprovação de que os dados analisados sigam uma determinada distribuição de probabilidade, pois o seu desconhecimento, e consequente má avaliação do risco poderia levar a um déficit na provisão das instituições financeiras.

A literatura acadêmica a respeito dos pressupostos sobre a distribuição de probabilidade para cômputo do VaR compreende as mais diversas abordagens. Assim, é possível citar trabalhos como Bali (2003) que propõe uma abordagem de distribuição de valores extremos para a estimativa do $\mathrm{VaR}$, demonstrando que a teoria estatística de extremos propicia estimativas mais corretas para a gestão do risco.

Seguindo também a teoria dos extremos, Danielsson e De Vries (2000) argumentam que as estimativas do VaR são muito dependentes de predições corretas a respeito de eventos incomuns e catastróficos, daí a importância de boas estimativas para as caudas das distribuições. Nesse contexto, os autores utilizam um método semi-paramétrico para a estimativa do VaR que é uma mistura dessas 
duas abordagens, onde se combinam simulação histórica não-paramétrica e estimação paramétrica das caudas da distribuição de retorno, a fim de estimar com precisão as caudas de uma distribuição.

Em Bhattacharyya et al. (2008) é possível encontrar uma revisão de estudos que aborda a questão da não normalidade dos retornos, mostrando que apesar dos esforços, as pesquisas estão longe de apontar para uma distribuição que se mostre mais adequada ao cômputo do VaR. O autor traz alguns exemplos como distribuição de $t$ de studet (BOLLERSLEV, 1987; HANSEN, 1994), a distribuição de erro generalizado (NELSON, 1991), a distribuição hiperbólica generalizada (EBERLEIN e KELLER, 1995; BARNDORFF-NIELSEN, 1997), distribuição estável (MCCULLOCH, 1996), a distribuição t não central (HARVEY e SIDDIQUE, 1999), distribuição GramCharlier (JONDEAU e ROCKINGER, 2001), distribuição Pearson Tipo IV (PREMARATNE e BERA, 2001; YAN, 2005), a distribuição t inclinada (JONDEAU e ROCKINGER, 2003), a distribuição de Johnson SU (YAN, 2005), e mistura de distribuições normais (ALEXANDER e LAZAR, 2006).

A despeito disso, não foi encontrado nenhum estudo que tratasse dos pressupostos de normalidade comparando carteiras grandes e diversificadas com ativos individuais, bem como o uso de critérios mais objetivos para a escolha da distribuição a ser considerada para o cálculo do VaR. Tal fato demonstra a contribuição incremental do trabalho, bem como a verificação do pressuposto de normalidade para a realidade do mercado de capitais nacional.

\subsection{Teste de Aderência de Kolmogorov-Smirnov}

Os testes de aderência são instrumentais estatísticos que permitem testar se duas amostras distintas são de uma mesma população, ou se a distribuição de probabilidade de uma série de dados pertence a uma distribuição de probabilidade teórica específica (DETTE et al., 2007). Este último caso possui uma aplicação interessante para as ferramentas de gestão de risco, dada a importância do conhecimento da correta distribuição de probabilidade.

Tais testes, usualmente, são baseados na função densidade empírica (EDF) e uma dada distribuição de probabilidade. Assim, testa-se a aderência de uma série de 
dados a uma distribuição de probabilidade específica utilizando distâncias entre essas duas curvas (D'AGOSTINO E STEPHENS, 1986).

Segundo Barbachan (2006), os principais testes de aderência baseados em distâncias e estatísticas EDF são o teste de Kolmogorov-Smirnov e o teste de Anderson-Darling. Nesses testes, a distância entre a EDF teórica e a EDF empírica é comparado a uma tabela de valores críticos para aceitar ou rejeitar a hipótese de que a amostra em questão (EDF empírica) pertence a uma determinada distribuição de probabilidade (EDF teórica).

Assim sendo, é efetuado o seguinte teste de hipótese:

$H O: F(x)=F O(x)$

$H 1: F(x) \neq F O(x)$

Onde, $F(x)$ a distribuição acumulada de uma amostra $x$, e $F 0(x)$ uma distribuição de freqüência acumulada teórica sob H0,

O teste de Kolmogorov-Smirnov a ser utilizado na presente investigação é um teste não paramétrico, ou seja, não necessita a admissão de normalidade e nível intervalar de mensuração (DEGROOT; SCHERVISH, 2002).

O procedimento básico para o teste procura especificar a distribuição de frequência acumulada que ocorreria sob a distribuição teórica, e compara-la com a distribuição de frequência acumulada observada. Sendo que a distribuição teórica representa o que se poderia observar sob $\mathrm{H}_{0}$. Assim, determina-se o ponto em que essas duas distribuições possuem a maior divergência, a qual se denomina $D_{\max }$. $A$ referência à distribuição amostral indica se essa diferencia máxima pode ser atribuída ao acaso. Isto é, a distribuição amostral indica se uma divergência de tal magnitude teria probabilidade de ocorrer se as observações constituíssem realmente uma mostra aleatória da distribuição teórica (SIEGEL, 1975). 


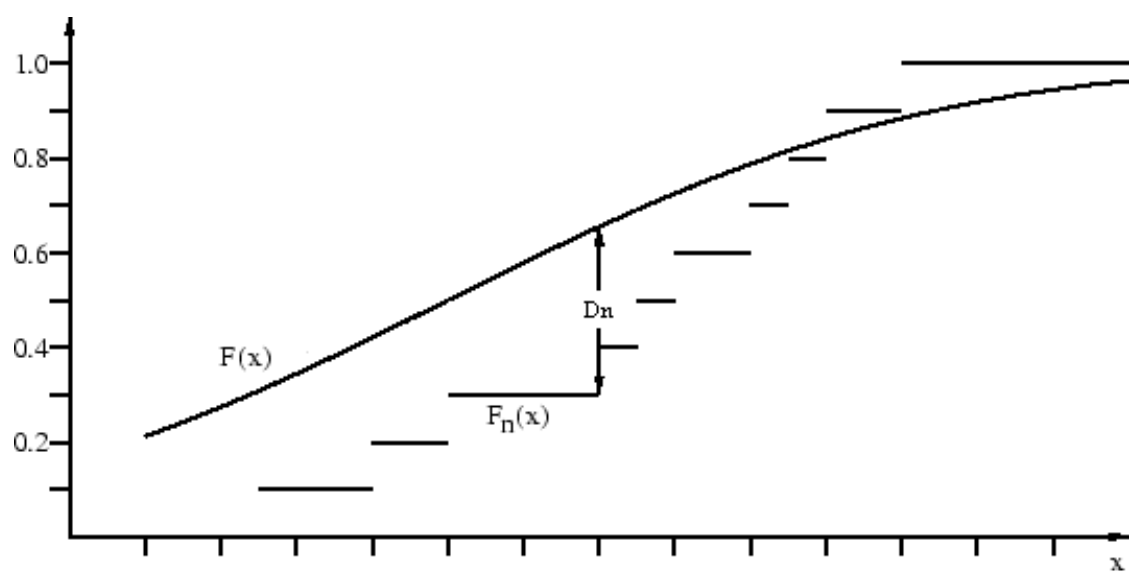

Fonte: http://members.cox.net/srice1/random/code.html (modificado pelo autor). Acesso em: $15 / 01 / 2010$

Portanto será efetuado o seguinte teste de hipóteses:

$H O: F(x)=F_{0}(x)$

$H 1: F(x) \neq F_{0}(x)$

Sendo $F(x)$ a distribuição acumulada de $x, F_{0}(x)$ uma distribuição de frequência acumulada teórica sob $H_{0}$, e seja $S_{N}(x)$ a distribuição de frequência acumulada de uma amostra aleatória de $\mathrm{N}$ observações, quando $x$ é qualquer escore possível, $S_{N}(x)=k / N$, onde $k$ é o número de observações não superiores a $x$ (THADEWALD E BÜNING, 2007).

Assim, sob a hipótese nula, espera-se que as diferenças entre $F_{0}(x)$ e $S_{N}(X)$ sejam pequenas e estejam dentro dos limites aleatórios. Esse desvio máximo $\left(D_{\max }\right)$ é dado pela maior diferença em valor absoluto, como pode ser observado em (4):

$\mathrm{D}=$ máximo $\left|F_{0}(X)-S_{N}(X)\right|$

Mediante os valores críticos, irá se determinar à probabilidade (bilateral) associada à ocorrência, sob $\mathrm{H}_{0}$, de valores tão grandes quanto o valor observado de D.

Para amostras maiores que 35 observações $(N>35)$, como de fato ocorreram no presente trabalho, utilizam-se os valores críticos para dez, cinco e um por cento (SIEGEL, 1973).

$$
\begin{aligned}
& \text { Para } \alpha=0,10 \Rightarrow D_{\text {critico }}=\frac{1,22}{\sqrt{N}} \\
& \text { Para } \alpha=0,05 \Rightarrow D_{\text {critico }}=\frac{1,36}{\sqrt{N}}
\end{aligned}
$$


Para $\alpha=0,01 \Rightarrow D_{\text {critico }}=\frac{0,63}{\sqrt{N}}$

Se $\left|D_{\text {calculado }}\right| \geq D_{\text {critico, } \alpha} \mid$, rejeita-se $\mathrm{H} 0$, concluindo que a amostra tem distribuição diferente da distribuição teórica testada.

\section{METODOLOGIA}

Para o experimento, foi coletada a cotação diária do índice IBOVESPA e de ações de 15 empresas individuais de capital aberto, selecionadas durante todos os dias de operação da Bolsa de valores de São Paulo no decorrer do ano de 2005. Tal ano foi escolhido por refletir um ambiente de relativa estabilidade econômica, propiciando melhores condições para a comparação da eficiência do pressuposto de normalidade para os ativos individuais e grandes carteiras.

Os dados foram coletados a partir da base ECONOMÁTICA ${ }^{\circledR}$, e o teste de Kolmogorov-Smirnov, bem como o cálculo do VaR foram realizados ao nível de significância de $5 \%$ a partir de uma rotina desenvolvida em MATLAB $7.1^{\circledR}$.

A escolha do índice IBOVESPA, advém do fato que as ações que compõem a carteira teórica deste correspondem a $80 \%$ do número de negócios e do volume financeiro do mercado à vista da BOVESPA, de modo a retratar da melhor forma possível o perfil dos negócios realizados na Bovespa. (ASSAF NETO, 1998; OLIVEIRA e REBELATTO, 2011). Nesse sentido, utiliza-se uma carteira ampla e diversificada para colocar à prova o pressuposto de normalidade.

As variações encontradas entre os dias representam os vários retornos diários (não foram considerados outros pagamentos como dividendos e bonificações) dos ativos individuais e do IBOVESPA. Os retornos diários compostos continuamente são calculados pela equação (5).

$$
R_{t}=\ln r_{t}-\ln r_{t-1}
$$

Onde $R_{t}$ é o retorno, $\ln r_{t}$ é o logaritmo na base neperiana que representa o valor no dia $t$, e $\ln r_{t-1}$ é o valor no dia $t-1$, o qual é amplamente usado em finanças e pesquisas acadêmicas como em Santos Filho et al. (2011).

Já para as distribuições que não admitem valores negativos em seus domínios, usou-se a expressão acima em termos de logaritmos, o que mantém a 
proporcionalidade percentual, mas somente com valores positivos de acordo com a equação (6).

$$
R p=\exp \left(R_{t}\right)
$$

Onde $R p$ são os retornos em termos exponenciais, e $R_{t}$ é resultado da equação (5). Nesse caso o VaR percentual será obtido pelo logaritmo natural de $R p$ na equação (6).

A partir disso, será montada uma distribuição de probabilidade acumulada empírica da carteira do IBOVESPA e das ações das empresas em questão para representar os respectivos retornos; tal distribuição empírica será comparada com diversas distribuições teóricas, tal qual é feito na FIGURA 3.

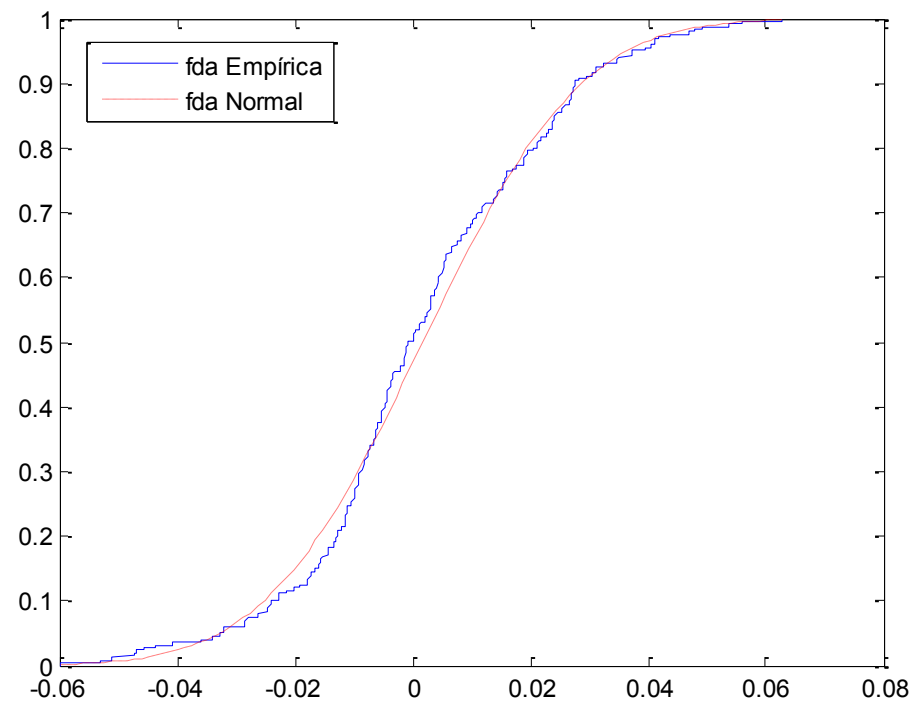

FIGURA 3 - Gráfico confeccionado em MATLAB® utilizando-se o estimador de Kaplan-Meyer (COX, D.R., e OAKES, D., 1984).

Com estes dados será conduzido um teste de Kolmogorov-Smirnov para uma amostra, que é uma prova de aderência. Isto é, diz respeito ao grau de concordância entre a distribuição de um conjunto de valores amostrais (observados) e determinada distribuição teórica específica (SIEGEL, 1975).

O teste de aderência foi realizado tanto para os retornos do índice IBOVESPA quanto para as ações das 15 empresas individuais; e foram verificadas aderências para as seguintes distribuições de probabilidade teóricas: Cauchy, Exponencial, Normal, Lognormal, Logística, Gama, Gumbel (valores extremos com cauda esquerda mais pesada), Pareto, Rayleigh e Weibull. 


\section{RESULTADOS E DISCUSSÕES}

Ao realizar os testes de aderência, muitos ativos aceitavam mais de uma distribuição de probabilidade, apesar de obterem p-valores distintos. Assim, a rotina desenvolvida em MATLAB ${ }^{B}$ resultava em um gráfico de aderência e uma tabela para cada distribuição não rejeitada no teste de Kolmogrov-Smirnov. Portanto, obteve-se para o IBOVESPA, por exemplo, nas Tabelas 1 e 2; enquanto os gráficos de aderência para a distribuição Normal e de Gumbel (valores extremos) são exibidos nas Figuras 4 e 5 via função densidade de probabilidade acumuladas.

Tabela 1 - Informações sobre o teste de aderência de Kolmogorov-Smirnov para a Distribuição Normal e de Valores Extremos para o índice IBOVESPA.

\begin{tabular}{ccc}
\hline & IBOVESPA & \\
\hline & $\boldsymbol{p}$-valor & VaR \\
\cline { 2 - 3 } Normal & 0,8670 & $-0,0246$ \\
Valores Extremos & 0,2825 & $-0,0351$
\end{tabular}

Na Tabela 1, por exemplo, é observável via p-valor que existe melhor aderência do portfólio IBOVESPA, à distribuição Normal; sendo observável, portanto, que a distribuição de valores extremos superestima o VaR.

Figura 4 - Gráfico de Aderência para distribuição Normal

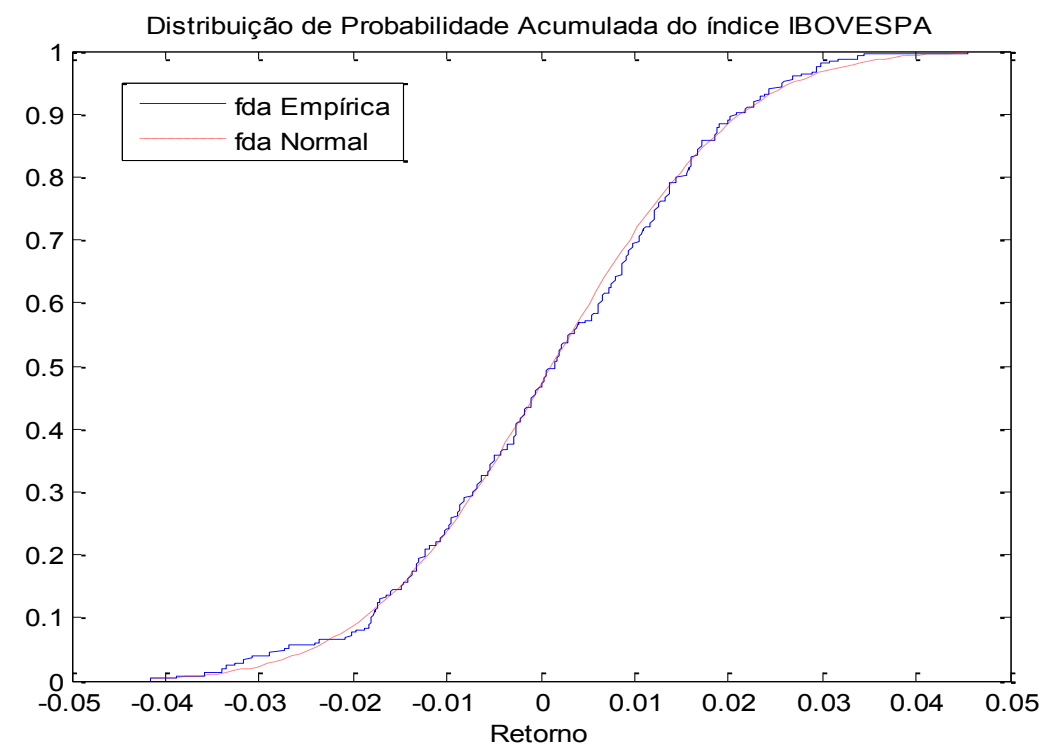

(Gráfico confeccionado em MATLAB®)

Revista Produção Online, Florianópolis, SC, v.14, n. 2, p. 430-447, abr./jun. 2014. 
Figura 5 - Gráfico de aderência para distribuição de valores extremos

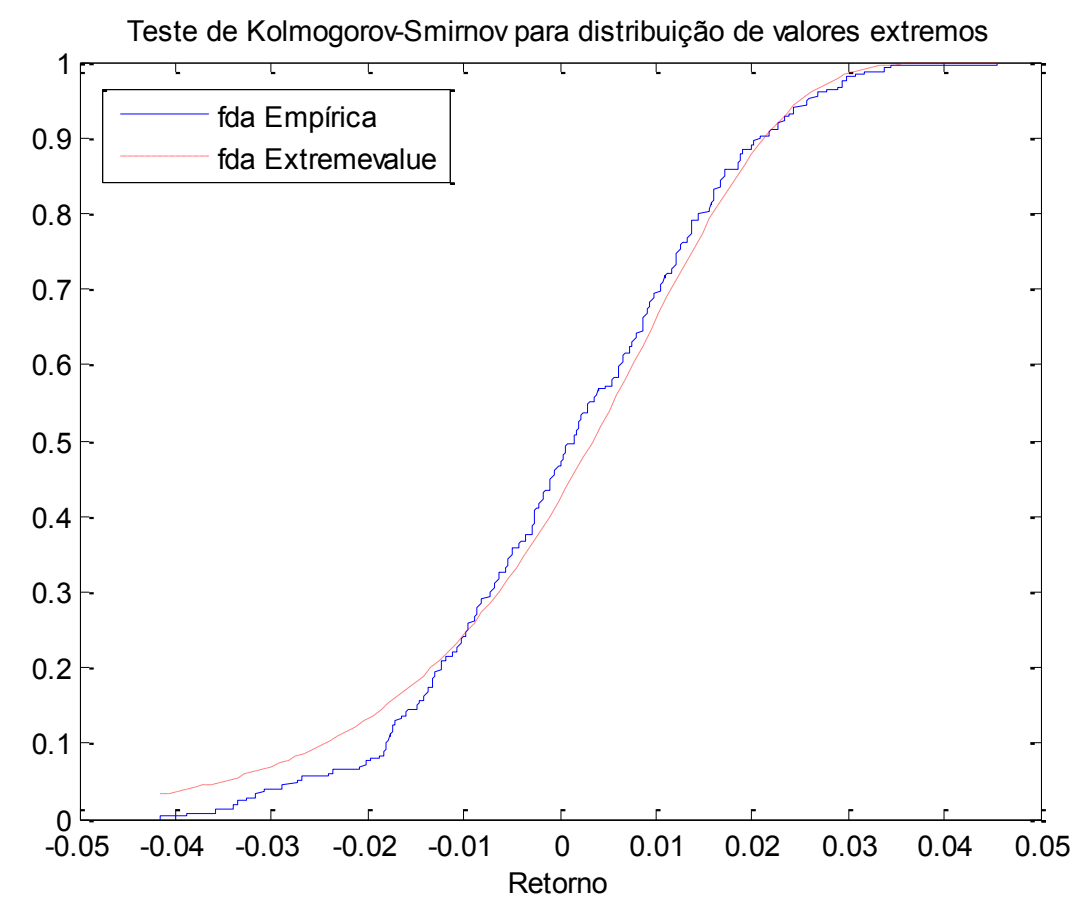

(Gráfico confeccionado em MATLAB®).

Desse modo, é notável que para cada ativo a rotina desenvolvida poderia gerar mais de um gráfico e uma tabela, fazendo com que para os 15 ativos individuais mais o índice IBOVESPA, resultassem em uma grande quantidade de informações que serão descritas de forma sucinta na Tabela 2.

Adiciona-se ainda que as Tabelas 1 e 2 apresentam uma informação indicada pelo " $p$-valor", a qual descreve a probabilidade da variável testada pertencer à distribuição teórica em questão. Portanto, quanto mais próximo da unidade for o $p$ valor, maior a chance do ativo testado pertencer à distribuição teórica proposta, visto que nesse caso para haver aderência requer-se que não seja rejeitada a hipótese nula. 
Tabela 2 - Resultados para os ativos individuais

\begin{tabular}{cccc}
\hline Ativo & $\begin{array}{c}\text { Melhor distribuição } \\
\text { encontrada }\end{array}$ & p-valor & VaR \\
\hline BBDC4 & Normal & 0,5985 & $-0,0314$ \\
PETR3 & Normal & 0,8491 & $-0,0288$ \\
VALE3 & Normal & 0,327 & $-0,0329$ \\
USIM5 & Normal & 0,9941 & $-0,0453$ \\
AMBV4 & Normal & 0,1316 & $-0,0241$ \\
PTIP4 & Gama & 0,7157 & $-0,0382$ \\
VCPA4 & Normal & 0,9777 & $-0,0336$ \\
BRKM5 & Gama & 0,8389 & $-0,0449$ \\
BRTO4 & Normal & 0,1472 & $-0,0512$ \\
EMBR3 & Gama & 0,7089 & $-0,0376$ \\
SDIA4 & Normal & 0,3258 & $-0,0380$ \\
CGAS5 & Normal & 0,2916 & $-0,0411$ \\
TMCP4 & Normal & 0,3682 & $-0,0399$ \\
ACES4 & Gama & 0,5163 & $-0,0413$ \\
CTA3 & Normal & 0,5933 & $-0,0388$ \\
& & & \\
\hline
\end{tabular}

$\mathrm{Na}$ amostra analisada, em todos os 15 ativos, juntamente com o índice IBOVESPA, houve rejeição das distribuições Cauchy, Logística e Pareto; diferentemente das outras distribuições testadas, as quais algumas vezes não puderam ser rejeitadas, mesmo com probabilidade baixa. Contudo, é observável que houve uma grande predominância das distribuições Normal e Gama com probabilidade e valores em risco muito próximos.

Para exemplificar o cálculo do VaR com aderência probabilística, pode-se utilizar o exemplo do valor em risco paramétrico calculado para o ativo da EMBRAER (EMBR3) na Tabela 2, no qual para a distribuição Normal foi obtido um $p$-valor = 0,6673 e um VaR de -0,0375; já para a distribuição Gama, encontrou-se um $p$-valor $=0,7089$ e um VaR de -0,0376. Portanto, a distribuição Gama apresentou uma melhor probabilidade de aderência para esse ativo.

Contudo, dado um lote de 1000 ações do ativo EMBRE3, com um preço de 15,21 reais por ação (por conveniência, o preço médio do ativo em 2005), obtém-se o valor total da posição de $15.210,00$ reais. Desta forma, o valor em risco da posição considerando-se uma distribuição Normal será de 570,357 reais (valor da posição 
multiplicado pelo VaR Normal); enquanto o VaR para a distribuição Gama, a qual demonstrou melhor aderência, será de 571,896 reais (valor da posição multiplicado pelo VaR Gama).

Tal diferença no cálculo do VaR ocorreu também com outros ativos como Braskem (BRKM5), onde para a distribuição Normal observou-se aderência de 0,8306 pelo p-valor e VaR correspondente a -0,04470; já para a distribuição Gama, a aderência foi de 0,8389 e o VaR igual a -0,04489. Aqui, para uma posição de 2.300.000,00 (100000 ações cotadas a 23 reais cada), observa-se um VaR de 102.810,00 reais para a distribuição Normal, e um VaR de 103.247,00 para a distribuição Gama, totalizando uma diferença de 437,00 reais. Tal valor pode ser considerado insignificante, tendo em vista a natureza probabilística do $\mathrm{VaR}$, e a magnitude da carteira em questão.

Assim, tal qual ocorrera na grande maioria dos ativos testados, houve pequena diferença na aderência Gama em relação à Normal, e na maioria dos casos observados, a diferença no VaR percentual ocorria na terceira ou quarta casa decimal, o que nos retornava valores em risco muito próximos mesmo usando diferentes distribuições de probabilidade.

\section{CONCLUSÕES}

Espera-se, a priori, que carteiras com muitos ativos fossem mais propensas a apresentar normalidade do que ativos individuais. Contudo em termos da aplicação do VaR paramétrico, tal expectativa mostrou-se aplicável também para ativos individuais, tendo em vista a realidade nacional.

Os resultados descritos demonstram que tanto para ativos individuais quanto para carteiras de grande porte, o pressuposto simplificador de normalidade mostrase aplicável, visto que as diferenças na provisão dos bancos (medida pelo VaR) era mínima quando utilizada uma distribuição com aderência ligeiramente melhor.

Contudo, o fato do pressuposto de normalidade mostrar-se eficiente na estimativa de risco não invalida o método proposto de cálculo do VaR paramétrico via teste de aderência, visto que tal experimento fora realizado em um ambiente de relativa estabilidade de mercados. Assim, tal instrumento pode ser eficaz para 
verificar pressupostos simplificadores, e, não obstante, servir de base para o cálculo do VaR em ambientes de instabilidade.

Enquanto alguns ativos individuais tiveram aderência satisfatória à distribuição Normal, em outros, o mesmo não ocorreu, de modo que não foi possível encontrar uma distribuição que se adequasse à modelagem dos retornos desses ativos dentre as 10 distribuições testadas. Tal fato demonstra que apesar de confiável na maioria dos casos, o VaR paramétrico com pressuposto de normalidade pode ser falho para alguns ativos, sendo necessária maior investigação no âmbito de outras distribuições de probabilidades alternativas ás aqui utilizadas.

Assim, os resultados demonstrados são válidos para um ambiente de estabilidade econômica, e, portanto, tais conclusões acerca da aplicabilidade do pressuposto de normalidade se mostrar eficiente tanto para ativos individuais quanto para grandes portfólios pode não ser aplicável num contexto de instabilidade e irracionalidade. Nesses termos, trabalhos futuros poderiam explorar tal questão por meio da comparação da eficiência do pressuposto de normalidade para ativos individuais e grandes carteiras tanto em épocas de crise quanto em períodos de estabilidade econômica.

É interessante salientar que a heterogeneidade dos dados de ativos de diferentes países pode explicar a variedade de distribuições de probabilidade consideradas adequadas para o cálculo do VaR na literatura. Assim sendo, os resultados encontrados no presente estudo aparentam ser válidos para a realidade brasileira; contudo, podem ser não extrapoláveis para outras conjunturas.

Estudos futuros também podem fazer uso de outros testes de aderência, no sentido de verificar se existem diferenças significativas entre os resultados obtidos com o teste de Kolmogorov-Smirnov e testes alternativos.

\section{AGRADECIMENTOS}

Os autores agradecem à FAPESP (Fundação de Amparo à Pesquisa do Estado de São Paulo) pelo apoio financeiro.

\section{REFERÊNCIAS}

ASSAF NETO, A. Mercado financeiro e suas aplicações. 4. ed. São Paulo: Atlas, 1998. 
AUSSENEGG, W., MIAZHYNSKAIA, T. Uncertainty in value-at-risk estimates under parametric and non-parametric modeling. Financial Markets and Portfolio

Management, v 20, n 3, p. 243-264, 2006.

http://dx.doi.org/10.1007/s11408-006-0020-8

BALI, T. G., An extreme value approach to estimating volatility and value at risk. The Journal of Business, v. 76, N. 1, p. 83-108, 2003. http://dx.doi.org/10.1086/344669

BARBACHAN, J. S. F., ORNELAS, J. R. H., FRIAS, A. R. Goodness-of-fit tests focuson value-at-risk estimation. Brazilian Review of Econometrics, v. 26, n 2, p. 309-326, 2006.

BHATTACHARYYA, M., CHAUDHARY A., YADAV G. Conditional VaR estimation using Pearson's type IV distribution. European Journal of Operational Research 191, p. 386-397, 2008. http://dx.doi.org/10.1016/j.ejor.2007.07.021

COX, D.R., OAKES D., Analysis of survival data. Chapman \& Hall, London, 1984.

D'AGOSTINO, R.B.; STEPHENS, M.A. Goodness-of-fit techniques. New York: Marcel Dekker, 1986.

DANIELSSON J., DE VRIES G. C., Value-at-risk and extreme returns , annales d'économie et de statistique, Microstructure des marchés financiers. Financial Market Microstructure, n. 60, p. 239-270, 2000.

DEGROOT, M. H., SCHERVISH, M. J. Probability and statistics. 3rd Edition. Addison Wesley, 2002.

DETTE, H.; NEUMEYER, N.; KEILEGOM, I. V., A new test for the parametric form of the variance function in non-parametric regression. Journal of the Royal Statistical Society. Series B (Statistical Methodology), v. 69 n. 5, p. 903-917, 2007. http://dx.doi.org/10.1111/j.1467-9868.2007.00616.x

ESCANCIANO, J. C., \& OLMO, J., Backtesting parametric value-at-risk with estimation risk. Journal of Business and Economic Statistics, v.28, n.1, p. 36-51, 2010. http://dx.doi.org/10.1198/jbes.2009.07063

GUJARATI, D. N. Econometria básica. 3.ed. São Paulo : Makron books, 2000.

JORION, P. Value at risk: the new benchmark for controlling derivatives risk. New York: McGraw Hill, 1997.

KAWATA, R.; KIJIMA, M. Value-at-risk in a market subject to regime switching. Quantitative Finance, v.7, n.6, 2007, p. 609 - 619.

http://dx.doi.org/10.1080/14697680601161795 
KHINDANOVA, I., RACHEV, S., SCHWARTZ, E. Stable modelling for value-at-risk. Mathematical and Computer Modelling, p. 1223-1259, 2001.

http://dx.doi.org/10.1016/S0895-7177(01)00129-7

KLEIBER, C., \& KOTZ, S. Statistical size distributions in economics and actuarial sciences. Wiley series in probability and statistics. Hoboken, New Jersey, 2003

MABROUK, S., SAADI, S., Parametric value-at-risk analysis: evidence from stock indices. Quarterly Review of Economics and Finance, 2012, p. 1-17.

MAREQUES, E. P., OLIVEIRA, F. A., BALESTRASSI, P. P. Quantificação da análise de riscos em investimentos usando medidas de dispersão. Revista Produção

Online, v. 1, n. 1, 2001. http://dx.doi.org/10.14488/1676-1901.v1i1.588

Oliveira, M. H. F., Rebelatto, D. A. N., Os Fundos de Investimentos em Ações no Mercado Financeiro Brasileiro: Os Gestores e Administradores são Capazes de Superar o Índice Bovespa? Revista Produção Online, v.11, n.1, 2011, p. 76-95. http://dx.doi.org/10.14488/1676-1901.v11i1.491

RAMANATHAN, R. Statistical methods in econometrics. San Diego: Academic Press, 1993.

SANTOS FILHO, E. L., SILVA, W. L., VEIGA, C. P., TORTATO, U., Previsão dos retornos do ibovespa utilizando redes neurais artificiais feedforward evolutivas. Revista Produção Online. Florianópolis, SC, v.11, n. 4, p. 1114-1140, 2011. http://dx.doi.org/10.14488/1676-1901.v11i4.784

SIEGEL, S. Estatística não-paramétrica. Rio de Janeiro: Editora McGraw-Hill, 1975.

SOUZA, L. A. R. Valor em risco em épocas de crise. Mimeo, tese de mestrado. São Paulo: FEA/USP, 1999.

THADEWALD, T.; BÜNING, H. Jarque-bera test and its competitors for testing normality: a power comparison. Journal of Applied Statistics, v. 34, n.1, 2007, p. 87 - 105. http://dx.doi.org/10.1080/02664760600994539

WANG, Z., WU, W., CHEN, C. AND ZHOU, Y. The exchange rate risk of chinese yuan: using var and es based on extreme value theory. Journal of Applied Statistics, v. 37, n. 2,2010 , p. $265-282$.

http://dx.doi.org/10.1080/02664760902846114

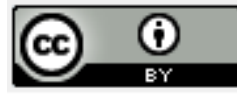

Artigo recebido em 27/10/2011 e aceito para publicação em 07/04/2014.

DOI: http://dx.doi.org/10.14488/1676-1901.v14i2.1130 